\title{
Probing the stepwise unfolding of bovine serum albumin using 2D Correlation Raman spectroscopy
}

Nikki Kuhar§, Siva Umapathy*,§,\#

§Department of Inorganic and Physical Chemistry, Indian Institute of Science, Bangalore, 560012, India \#Department of Instrumentation \& Applied Physics, Indian Institute of Science, Bangalore, 560012, India

CORRESPONDING AUTHOR:* siva.umapathy@gmail.com

Table of content

S.1. Details about Amide I curve fitting

S.2. Details about 2D Correlation analysis

S.3. References

S.4. Supplementary Table S-1

S.5. Supplementary Table S-2

S.6. Supplementary Figure S-1

S.7. Supplementary Figure S-2

S.8. Supplementary Figure S-3

S.9. Supplementary Figure S-4 
S.1. Amide I curve fitting. Amide I band is broadband which ranges from $1580 \mathrm{~cm}^{-1}$ to $1700 \mathrm{~cm}^{-1}$. The amide I band is a complex, unresolved line shape where the maximum is accompanied by various shoulders assigned for various secondary structures. In order to identify and characterize the main component bands underneath the amide I profile, curve fitting is usually performed . ${ }^{1,2}$ To perform the curve fitting, amide band was considered from $1550 \mathrm{~cm}^{-1}$ to $1720 \mathrm{~cm}^{-1}$ to avoid fitting errors at the corners. A linear baseline is assumed for this region. The initial position, height and bandwidth of the bands used for fitting were determined from the second derivative spectra. There were two extra peaks before $1600 \mathrm{~cm}^{-1}$ and one peak after $1720 \mathrm{~cm}^{-1}$ were also used for fitting apart from amide I peaks as shown by dotted lines in figure 3(B). However, these peaks are not analysed. The position of the band maximum remains the same in the second derivative. The height of a second-derivative peak is proportional to the square of the original peak height with an opposite sign while the half bandwidth is inversely proportional to the square of the original half-bandwidth ${ }^{3,4}$ The second-derivative spectra were calculated using the Savitzky-Golay derivative algorithm as shown in figure S-1. The resulting spectra were smoothed by a 9-point Savitsky-Golay function, to improve the quality of the data. The spectra were fitted using Renishaw Wire 3.2 (Renishaw Inc. UK) software. Lorentzian line shapes were assigned for various peaks. During the fitting process, the height, width and positions of all bands were varied simultaneously. The iterations were repeated unless best fit was achieved as determined by minimum reduced $\chi^{2}$ values.

S.2. 2D Correlation analysis. Two-dimensional correlation spectroscopy (2D COS) is a mathematical technique to investigate the behavior of a system in response to external perturbations which can be temperature, $\mathrm{pH}$, concentration, time etc..$^{5-8}$ A set of spectra are measured after a systematic perturbation which is a change in $\mathrm{GuHCl}$ in the present study. A dynamic spectrum is constructed by subtracting a reference spectrum. The dynamic spectrum constitutes the changes in intensity and bad shifts over the perturbation. 2DCoS quantitatively compares the spectral intensity at two wavenumbers $v_{1}$ and $v_{2}$ along with perturbation. A dynamic spectrum is analyzed using 2D Shige software which generates synchronous and asynchronous graphs. The synchronous spectrum gives 'in phase' information i.e. which peaks are changing simultaneously. While asynchronous spectrum gives 'out of phase' information i.e. relative order of events (which peak is changing first compared to other). It spreads the spectrum over two frequency axes while spectral intensity is plotted as third coordinate. The resolution of the system is improved due to the spreading of highly overlapped peaks along the second dimension. 


\section{S.3. References}

(1) Maiti, N. C.; Apetri, M. M.; Zagorski, M. G.; Carey, P. R.; Anderson, V. E. Raman Spectroscopic Characterization of Secondary Structure in Natively Unfolded Proteins: a-Synuclein. J. Am. Chem. Soc. 2004, 126 (8), 2399-2408.

(2) Berjot, M.; Marx, J.; Alix, A. J. P. Determination of the Secondary Structure of Proteins from the Raman Amide I Band: The Reference Intensity Profiles Method. $J$. Raman Spectrosc. 1987, 18 (4), 289-300.

(3) Byler, D. M.; Susi, H. Examination of the Secondary Structure of Proteins by Deconvolved FTIR Spectra. Biopolymers 1986, 25 (3), 469-487.

(4) Dong, A.; Huang, P.; Caughey, W. S. Protein Secondary Structures in Water from Second-Derivative Amide I Infrared Spectra. Biochemistry 1990, 29 (13), 3303-3308.

(5) Jung, Y. M.; Noda, I. Two-Dimensional Correlation Spectroscopy: New Developments and Applications. In Encyclopedia of Analytical Chemistry; John Wiley \& Sons, Ltd: Chichester, UK, 2018; pp 1-27.

(6) Noda, I.; Liu, Y.; Ozaki, Y. Two-Dimensional Correlation Spectroscopy Study of Temperature-Dependent Spectral Variations of W-Methylacetamide in the Pure Liquid State. 1. Two-Dimensional Infrared Analysis. J. Phys. Chem. 1996, 100 (21), 86658673.

(7) Noda, I.; Ozaki, Y. Two-Dimensional Correlation Spectroscopy - Applications in Vibrational and Optical Spectroscopy; 2005.

(8) Noda, I.; Ozaki, Y. Two-Dimensional Correlation Spectroscopy - Applications in Vibrational and Optical Spectroscopy; John Wiley \& Sons, Ltd: Chichester, UK, 2004.

\section{S.4. Supplementary Table}

Table S-1: Frequencies and assignment of Raman bands of BSA in water

\begin{tabular}{ll}
\hline Wavenumber $\left(\mathrm{cm}^{-1}\right)$ & Assignment \\
\hline $\mathbf{1 5 8 0}, 1606,1615,1630$ & Side chain residues \\
\hline
\end{tabular}




\begin{tabular}{ll}
\hline $1635-1640$ & Short-segment chains connecting the $\alpha$ helical segment \\
$1650-1658$ & $\alpha$ helix \\
$1660-1670$ & $\beta$ sheets \\
$1670-1680$ & $\beta$ turns \\
$1680-1690$ & Random Structure \\
1700 and above & hydrogen bonded (weak, medium, strong) COOH \\
\hline
\end{tabular}

\section{S.5. Supplementary Table}

Table S-2: Signs of synchronous $(\Phi)$ and asynchronous $(\Psi)$ cross peaks and sequential order for amide III region in the concentration range of $\mathrm{GuHCl}$ from $2 \mathrm{M}$ to $4 \mathrm{M}$. (a>b implies a occurs before b)

\begin{tabular}{|c|c|c|c|c|}
\hline & $\begin{array}{c}\text { Cross Peak } \\
(\text { wavenumber cm }\end{array}$ & $\Phi\left(v_{1}, v_{2}\right)$ & $\Psi\left(v_{1}, v_{2}\right)$ & Sequential order \\
\hline 1 & 1303,1253 & - & + & $1253>1303$ \\
\hline 2 & 1303,1243 & - & + & $1243>1303$ \\
\hline 3 & 1303,1236 & - & + & $1236>1303$ \\
\hline 4 & 1252,1243 & + & - & $1243>1252$ \\
\hline 5 & 1252,1236 & + & - & $1236>1252$ \\
\hline 6 & 1243,1237 & + & - & $1236>1243$ \\
\hline
\end{tabular}

\section{S.6. Supplementary Figure}




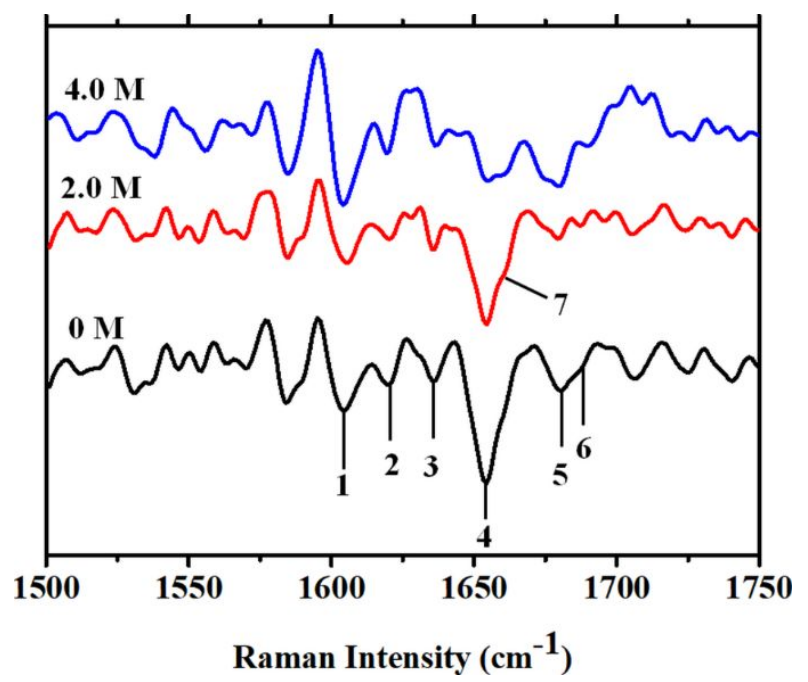

Figure S-1. Second derivate spectra of BSA at $0,2.0$ and 4.0 M concentration of GuHCl. Second derivate spectra have been calculated using Savitzky-Golay algorithm and smoothed.

\section{S.7. Supplemetary Figure}

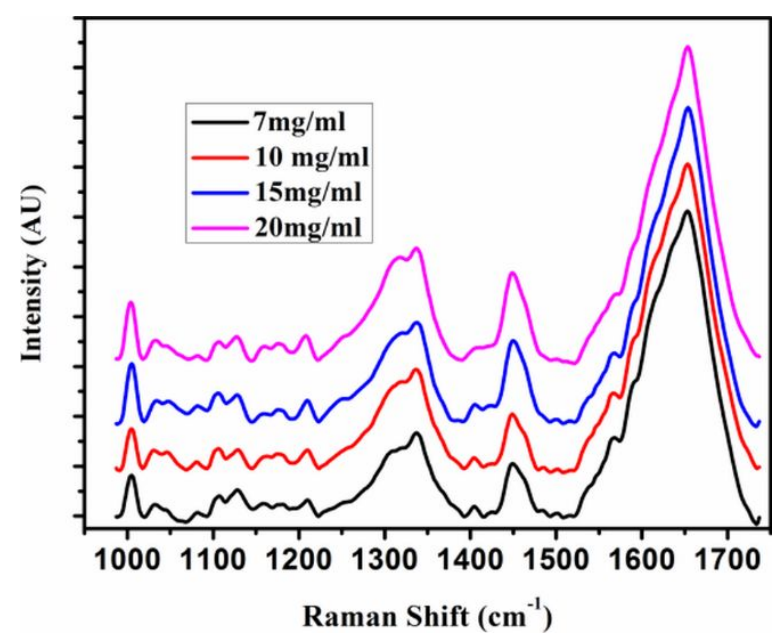

Figure S-2. Pre-processed Raman spectra of BSA dissolved in buffer at various concentration. All spectra have been corrected for cosmic rays, background and vectornormalized 
S.8. Supplemetary Figure

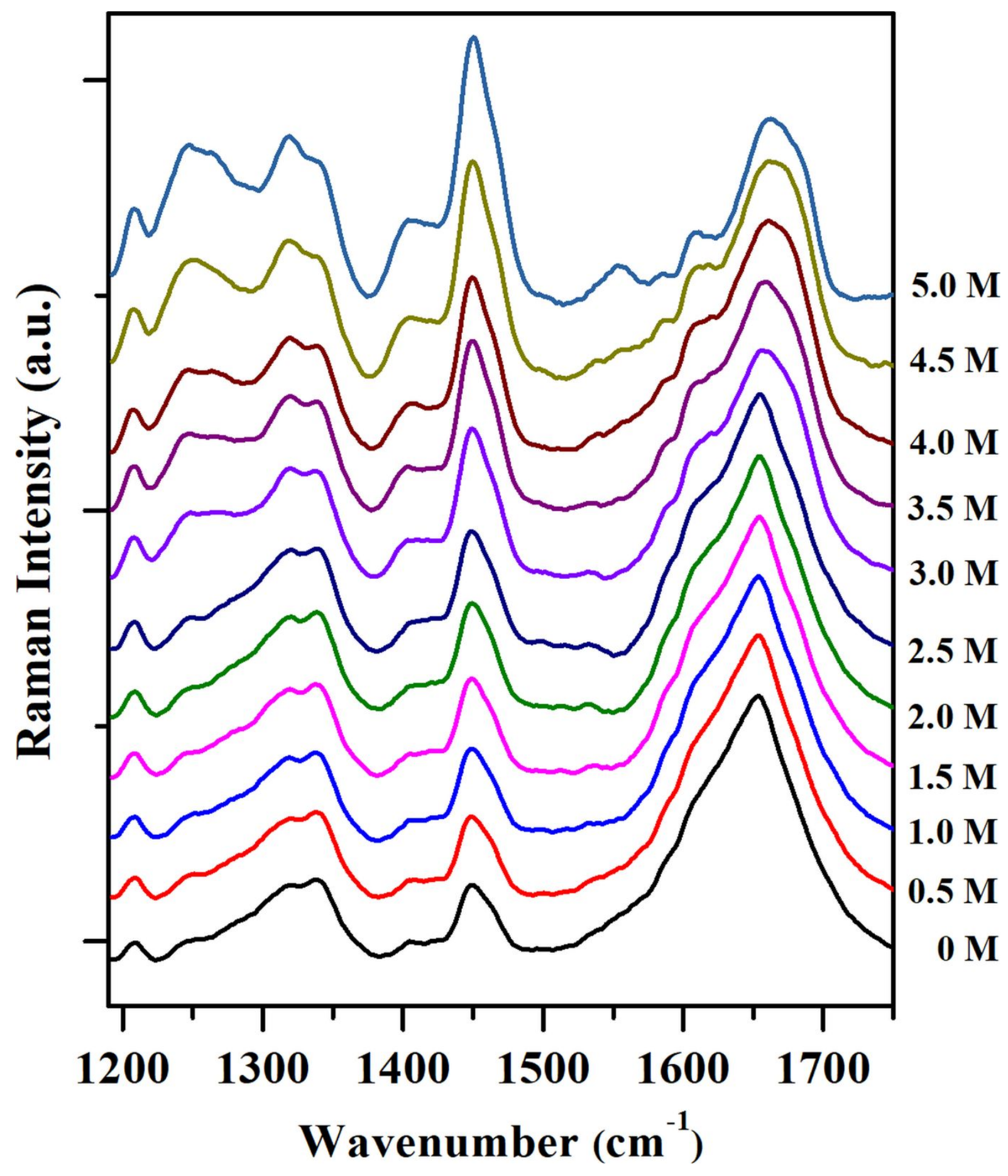

Figure S-3. Background corrected Raman spectra of BSA under the various concentration of $\mathrm{GuHCl}$ 
S.9. Supplemetary Figure

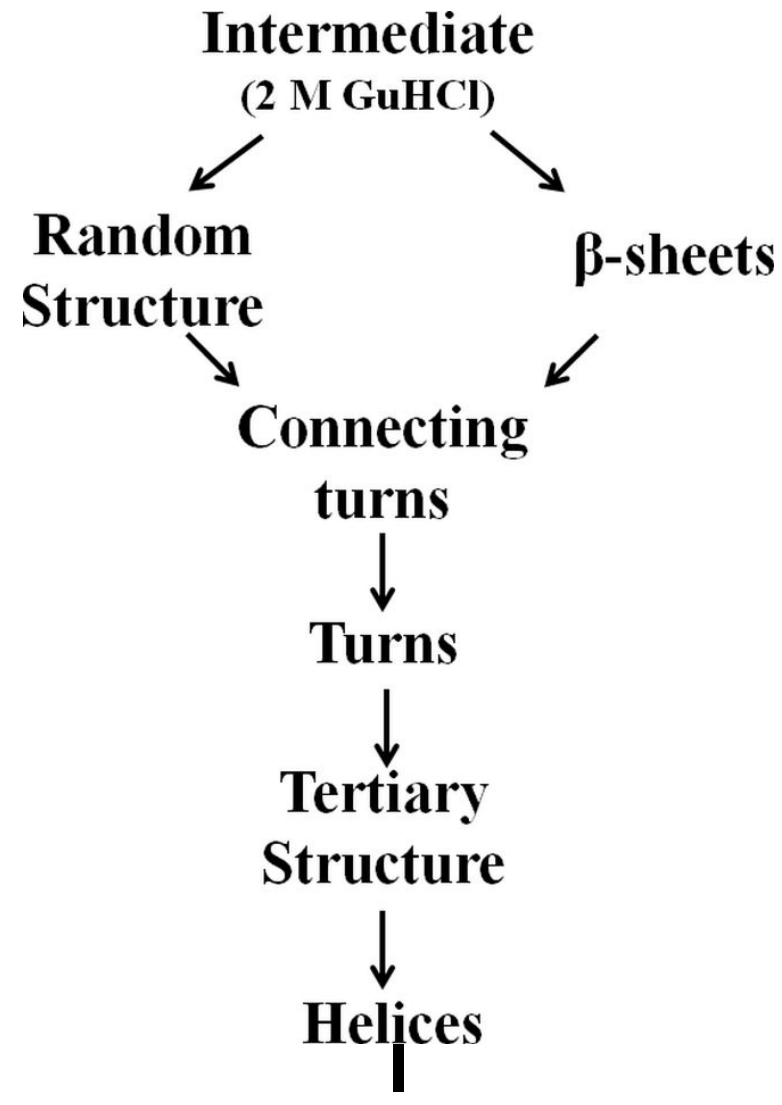

Figure S-4. Sequence of the evolution of various secondary structures during denaturation of BSA deduced using 2D Correlation analysis of amide I band 